\title{
S100A12 on Circulating Endothelial Cells Surface in Children With Kawasaki Disease
}

\author{
SONGLING FU, FANGQI GONG, CHUNHONG XIE, WEIHUA ZHU, WEI WANG, HONGQIANG SHEN, AND YONGMIN TANG
}

Department of Cardiology [S.F., F.G., C.X., W.Z.] and Department of Hematology/Oncology [H.S., Y.T.], Children's Hospital, Zhejiang University School of Medicine, Hangzhou, Zhejiang 310003, China

\begin{abstract}
The purpose of this study was to investigate the expression of S100A12 on the surface of circulating endothelial cells (CECs) in children with Kawasaki disease (KD) and the correlations between S100A12 and coronary artery lesions (CALs). The ratio of CECs to mononuclear cells (CECs/MNC), the positive rate of S100A12 on CECs surface (CECs-S100A12/CECs), and the fluorescence intensity of S100A12 on CECs surface (FI-S100A12-CECs) were evaluated respectively in 42 patients with acute stage (A-KD), subacute stage (SA-KD) and convalescent stage $\mathrm{KD}$ (C-KD). The $\mathrm{CECs} / \mathrm{MNC}$ ratio increased significantly in patients with A-KD and SA-KD with CALs. The CECs-S100A12/CECs rate and FIS100A12-CECs level were significantly higher in patients with KD than in the controls. The FI-S100A12-CECs level decreased to near half levels in patients with SA-KD and C-KD without CALs, but increased continuously in patients with SA-KD with CALs. The CECs/MNC ratio and FI-S100A12-CECs level in patients with SA-KD with CALs were significantly higher than in patients with SA-KD without CALs. The FI-S100A12-CECs level was significantly higher in patients with C-KD with CALs than in C-KD without CALs. The S100A12 expression on the CECs surface increased significantly in patients with KD and persisted for a longer time in patients with CALs, suggesting that the S100A12 expression on CECs may be involved in the development of CALs. (Pediatr Res 68: 165-168, 2010)
\end{abstract}

$\mathrm{K}^{2}$ awasaki disease (KD), an acute febrile disease that usually occurs in children under 5 years of age (1), has become the primary cause of acquired heart disease in children (2) (3). KD affects predominantly small and mediumsized arteries, particularly the coronary artery (4), with potential risks for coronary stenosis and thrombosis caused by coronary artery lesions (CALs), and even death caused by myocardial infarction (5) (6). The CALs can be found in $\sim 10-15 \%$ of children with KD (7). Although the treatment with i.v. immunoglobulin (IVIG) for patients with acute stage $\mathrm{KD}(\mathrm{A}-\mathrm{KD})$ can reduce the duration of fever and the incidence of CALs, $\sim 10 \%$ of children with KD do not respond to IVIG treatment with higher risk for CALs (8) (9). The cause and mechanisms of CALs development remain unclear (10).

Endothelial damage is the basic pathologic process in the development of many diseases. Circulating endothelial cells (CECs) is a new sensitive indicator for studying vascular endothelial function in vivo. It was first proposed by Bouvier

Received January 5, 2010; accepted April 28, 2010.

Correspondence: Fangqi Gong, M.D., Ph.D., Children's Hospital, Zhejiang University School of Medicine, Hangzhou, Zhejiang, 310003 China; e-mail: gongfangqi@zju.edu.cn

Supported by the National Nature Scientific Research Foundation [30872799]; and the Zhejiang Nature Scientific Research Foundation [Y207201] in China. in the 1970s that CECs might be regarded as an indicator of vascular injury. Some data have shown that the quantitative changes of CECs could help to evaluate the health status of endothelial cells and to assess the cardiovascular risk (11). CALs caused by KD are closely related to the function of coronary artery endothelial cells, and the CEC level increases significantly in the blood of patients with acute KD (12).

S100A12 expresses normally in neutrophils, whereas low in lymphocytes and monocytes. The extracellular S100A12 protein may activate many signal transduction pathways, including phospholipase $\mathrm{C}$ (PLC), protein kinase $\mathrm{C}$ (PKC), calmodulin kinase II, and mitogen activated protein kinase (13) (14). Many studies showed that S100A12 protein has a close relation with KD. Ebihara et al. (15) examined the expression levels of S100A12 protein genes in leukocyte by quantitative real-time PCR in the patients with acute and convalescent stage KD (C-KD). Significantly elevated expression of S100A12 genes was observed in patients with A-KD. Foell et al. (16) found that the S100A12 protein expression increased significantly in the serum of patients with A-KD, but decreased quickly in response to IVIG therapy, indicating S100A12 may be used as a markers of the activity of KD. It was found that S100A12 is actively secreted by neutrophils in patients with active KD by flow cytometry analysis in peripheral blood (17). The correlation between S100A12 protein expression and KD remains unclear, and the role of S100A12 in KD has not yet been fully defined.

There has been no report on the expression of S100A12 protein on the CECs surface in patient with KD. To make sure whether there is relationship between KD and S100A12 protein on the CECs surface, we investigated the ratio of CECs to mononuclear cells (CECs/MNC), the positive rate of S100A12 on the CECs surface (CECs-S100A12/CECs), and the fluorescence intensity of S100A12 on the CECs surface (FI-S100A12$\mathrm{CECs}$ ) by MAb combined with flow cytometry in KD children with or without CALs in acute, sub-acute, and convalescent stages.

\footnotetext{
Abbreviations: A-KD, KD in acute stage; C-KD, KD in convalescent stage; CALs, coronary artery lesions; CECs, circulating endothelial cells; CECs/ MNC, the ratio of CECs to mononuclear cells; CECs-S100A12/CECs, the positive rate of S100A12 on CECs surface; FI-S100A12-CECs, the fluorescence intensity of S100A12 on CECs surface; IVIG, intravenous immunoglobulin; KD, Kawasaki disease; SA-KD, KD in subacute stage
} 


\section{MATERIALS AND METHODS}

The study was approved by the ethical committee of the Children's Hospital, Zhejiang University School of Medicine, and was based on the institution's guidelines for human studies. Informed consent was obtained from all the subjects.

Subjects. Forty-two patients with KD (26 boys and 16 girls), aged 3-132 mo $(32.2 \pm 23.8 \mathrm{mo})$, were enrolled. The duration of fever was 3-11 d (6.2 \pm $3.1 \mathrm{~d})$. The erythrocyte sedimentation rate and C-reactive protein were $62 \pm$ $33 \mathrm{~mm} / \mathrm{h}$ and $72 \pm 41 \mathrm{mg} / \mathrm{L}$ in A-KD and $57 \pm 35 \mathrm{~mm} / \mathrm{h}$ and $21 \pm 20 \mathrm{mg} / \mathrm{L}$ in sub-acute stage (SA-KD).

All the patients were treated with IVIG at $1 \mathrm{~g} / \mathrm{kg} / \mathrm{d}$ for $2 \mathrm{~d}$ and oral aspirin at $30-50 \mathrm{mg} / \mathrm{kg} / \mathrm{d}$. After $3-5 \mathrm{~d}$ of treatment when the patients' temperature returned to normal, the dose of aspirin was reduced to $3-5 \mathrm{mg} / \mathrm{kg} / \mathrm{d}$ for $12 \mathrm{wk}$. There were six IVIG-resistant patients with KD whose temperature was still higher than $38^{\circ} \mathrm{C}$ after $48 \mathrm{~h}$ of standard treatment, then they were continued with IVIG at $1 \mathrm{~g} / \mathrm{kg} / \mathrm{d}$ for another $2 \mathrm{~d}$. All the patients with KD fulfilled the diagnostic criteria established by the MCLS research committee of Japan (2002) (18)

Ten patients ( 6 boys and 4 girls), aged 6 to 112 mo (30.2 \pm 22.4 mo), were found with CALs by echocardiography during the disease process. Coronary artery lesion (CAL) was defined as the internal lumen diameter $>2.5 \mathrm{~mm}$ in children $<3$ y of age, $>3 \mathrm{~mm}$ in children $3-9 \mathrm{y}$ of age, $>3.5 \mathrm{~mm}$ in children $9-14 \mathrm{y}$ of age; the internal diameter of a segment measured $\geq 1.5$ times that of an adjacent segment; and the lumen was clearly irregular.

The disease process duration between $4-10 \mathrm{~d}, 11-21 \mathrm{~d}$, and 22-60 $\mathrm{d}$ was defined as A-KD, SA-KD, and C-KD, respectively.

Sixty healthy children (38 boys and 22 girls), aged $4-120$ mo (33.4 \pm 21.8 mo), were enrolled in the control group.

Blood samples. Two milliliters of venous blood samples were collected in patients with A-KD before IVIG treatment, patients with SA-KD, patients with C-KD, and healthy children and anticoagulated with 1:10 heparin. Anticoagulated blood samples were kept at $4{ }^{\circ} \mathrm{C}$ and analyzed by flow cytometry within $2 \mathrm{~h}$ from venesection. The samples were divided into two tubes (each with $100 \mu \mathrm{L}$ ): one tube was used for testing by adding $10 \mu \mathrm{L}$ polyclonal goat anti-human S100A12 antibody (R\&D systems), and the other was used as control by adding goat IgG1. After $30 \mathrm{~min}$, both tubes were washed once using PBS, then incubated with FITC-labeled rabbit anti-goat IgG (USA, Southern Biotech) for $30 \mathrm{~min}$. After washing twice with PBS and removing the supernatant, the control tube was stained with $5 \mu \mathrm{L}$ CD45PerCP MoAb (CD45 MAb was conjugated with PERidinin-chlorophyll- $\alpha$ protein, USA Bcton Dickinson) and mouse IgG1-PE, whereas the testing tube was stained with $5 \mu \mathrm{L}$ CD45-PerCP MoAb and $5 \mu \mathrm{L}$ CD146-PE MoAb (CD146 MAb was conjugated with R-phycoerythrin, USA Bcton Dickinson). Incubated at $4{ }^{\circ} \mathrm{C}$ for $20 \mathrm{~min}$, the two tubes were added with $400 \mu \mathrm{L}$ hemolytic agent (FACSTM, USA Bcton Dickinson) for $10 \mathrm{~min}$, and centrifuged at 1000 $\mathrm{rpm}$ for $10 \mathrm{~min}$. Finally, the two tubes were added with $500 \mu \mathrm{L}$ PBS and mixed well to be detected after removal of the supernatant.

Flow cytometry. The samples in both tubes were evaluated by flow cytometry (FACS Calibur, USA Bcton Dickinson). Flow cytometry data were analyzed with CellQuest software. A multistep manual technique was used to detect and quantify the CECs and S100A12 on CECs surface. One hundred thousand cells were detected including MNC (lymphocytes, monocytes, and CECs) and polymorphonuclear leukocytes, and data were kept in the computer. Excitation light sources were $488 \mathrm{~nm}$ for FITC, PE, and PerCP.

Gating and quantification of CECs and S100A12 on CECs surface. The first step was to screen MNC (R1) (Fig. 1A), and the second step was to screen CECs (Fig. 1B). CECs were identified if the cells were CD146+CD45-cells (19). A region (R2) of CD146+CD45-cells with known light scatter properties was labeled CECs. The third step was to verify the CECs again (R3) (Fig. $1 C$ ) and to calculate the ratio of CECs to MNC (CECs/MNC). The last step was to measure the positive rate of S100A12 on CECs surface (CECsS100A12/CECs) and the fluorescence intensity of S100A12 on CECs surface (FI-S100A12-CECs) (Fig. 1D).

Statistical analysis. Analysis of the difference between the groups of patients was accomplished using ANOVA followed by LSD multiple comparison for normal data, whereas Mann-Whitney test was applied to analyze nonparametric data. The difference was considered significant with a $p$ value $<0.05$. The results were expressed as mean $\pm \mathrm{SD}$. All analysis was implemented in SPSS 16.0 software.

\section{RESULTS}

CECs/MNC, CECs-S100A12/CECs, and FI-S100A12CECs levels in children with $K \boldsymbol{D}$. The $\mathrm{CECs} / \mathrm{MNC}$ ratio was significantly $(p<0.05)$ higher in patients with A-KD and
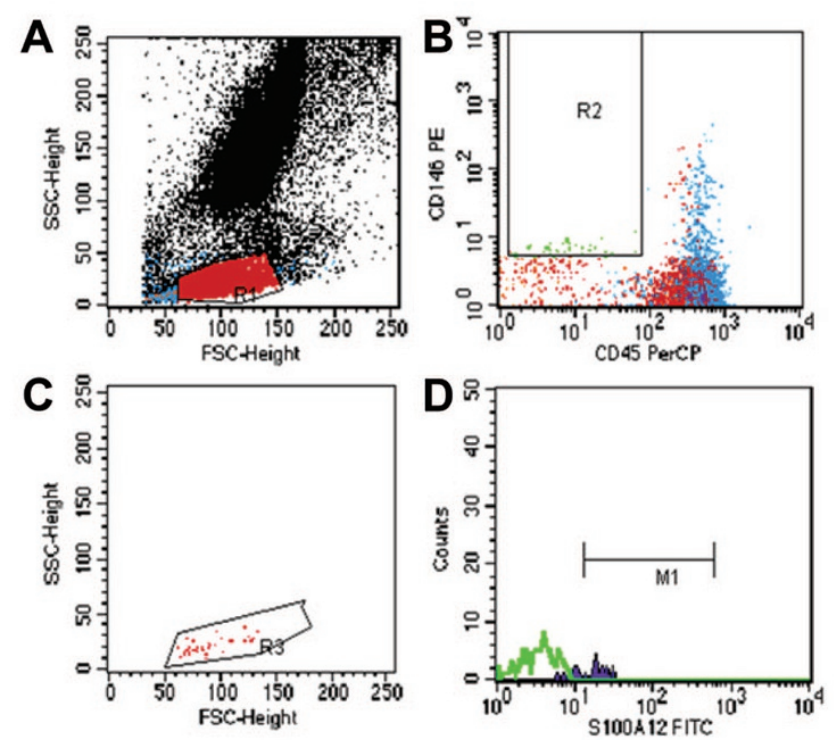

Figure 1. Diagram of CECs/MNC, CECs-S100A12/CECs, and FIS100A12-CECs measurement by flow cytometry. A, screening MNC (R1); $B$, screening CECs (R2); $C$, verifying the CECs again (R3); $D$, measuring CECs-S100A12/CECs and FI-S100A12-CECs, the green represent the isotype control scan.

Table 1. CECs/MNC, CECs-S100A12/CECs, and FI-S100A12-CECs in children with Kawasaki disease

\begin{tabular}{lcccc}
\hline & & $\begin{array}{c}\text { CECs/MNC } \\
(\%)\end{array}$ & $\begin{array}{c}\text { CECs-S100A12/ } \\
\text { CECs }(\%)\end{array}$ & FI-S100A12-CECs \\
\hline A-KD & 42 & $1.29 \pm 1.08^{*}$ & $63 \pm 16^{*}$ & $213 \pm 256^{*}$ \\
SA-KD & 42 & $1.19 \pm 1.29^{*}$ & $59 \pm 24^{*}$ & $264 \pm 270^{*}$ \\
C-KD & 42 & $0.71 \pm 0.63 \dagger$ & $57 \pm 16^{*}$ & $128 \pm 143^{*} \dagger$ \\
Control & 60 & $0.69 \pm 0.50$ & $38 \pm 16$ & $61 \pm 50$ \\
\hline
\end{tabular}

$* p<0.05$ compared with control.

$\dagger p<0.05$ compared with A-KD and SA-KD.

CECs/MNC, the ratio of circulating endothelial cells to mononuclear cells; CECs-S100A12/CECs, the positive rate of S100A12 on the surface of circulating endothelial cells; FI-S100A12-CECs, the fluorescence intensity of S100A12 on the surface of circulating endothelial cells.

SA-KD than in controls, and decreased to near normal levels in patients with C-KD. The CECs-S100A12/CECs rate and FI-S100A12-CECs levels were significantly $(p<0.05)$ higher in patients with A-KD, SA-KD, and C-KD than in controls. The CECs-S100A12/CECs rate did not decrease significantly in patients with SA-KD and C-KD. Although the FIS100A12-CECs level decreased significantly in patients with $\mathrm{C}-\mathrm{KD}$, it was still higher than the level in controls (Table 1).

CECS/MNC, CECs-S100A12/CECs, and FI-S100A12CECs levels in KD without CALs. The CECs/MNC ratio was significantly $(p<0.05)$ higher in patients with A-KD without CALs than in controls, and decreased to near normal levels in patients with SA-KD and C-KD. The CECs-S100A12/CECs rate and FI-S100A12-CECs levels were significantly $(p<$ 0.05 ) higher in patients with A-KD, SA-KD, and C-KD without CALs than in controls. The CECs-S100A12/CECs levels did not decrease significantly in patients with SA-KD and C-KD without CALs. The FI-S100A12-CECs levels decreased significantly in patients with SA-KD and C-KD with- 
out CALs but were still higher than the level in controls (Table 2).

CECs/MNC, CECs-S100A12/CECs, and FI-S100A12CECs levels in $\mathbf{K D}$ with CALs. The CECs/MNC ratio was significantly $(p<0.05)$ higher in patients with A-KD and SA-KD with CALs than in controls, and decreased to near normal levels in patients with C-KD. The CECs-S100A12/ CECs rate and FI-S100A12-CECs levels were significantly $(p<0.05)$ higher in patients with A-KD, SA-KD, and C-KD

Table 2. CECs/MNC, CECs-S100A12/CECs, and FI-S100A12-CECs in patients with KD without CALs

\begin{tabular}{lcccc}
\hline & & $\begin{array}{c}\text { CECs/MNC } \\
(\%)\end{array}$ & $\begin{array}{c}\text { CECs-S100A12/ } \\
\text { CECs (\%) }\end{array}$ & FI-S100A12-CECs \\
\hline $\begin{array}{c}\text { A-KD without } \\
\text { CALs }\end{array}$ & 32 & $1.29 \pm 0.97^{*}$ & $61 \pm 15^{*}$ & $208 \pm 267^{*}$ \\
$\begin{array}{c}\text { SA-KD without } \\
\quad \text { CALs }\end{array}$ & 32 & $0.78 \pm 1.04 \dagger$ & $58 \pm 23^{*}$ & $108 \pm 89^{*} \dagger$ \\
$\begin{array}{c}\text { C-KD without } \\
\quad \text { CALs }\end{array}$ & 32 & $0.68 \pm 0.47 \dagger$ & $57 \pm 13^{*}$ & $102 \pm 65^{*} \dagger$ \\
Control & 60 & $0.69 \pm 0.50$ & $38 \pm 16$ & $61 \pm 50$ \\
\hline
\end{tabular}

$* p<0.05$ compared with control.

$\dagger p<0.05$ compared with A-KD.

$\mathrm{CECs} / \mathrm{MNC}$, the ratio of circulating endothelial cells to mononuclear cells; CECs-S100A12/CECs, the positive rate of S100A12 on the surface of circulating endothelial cells; FI-S100A12-CECs, the fluorescence intensity of S100A12 on the surface of circulating endothelial cells.

Table 3. CECs/MNC, CECs-S100A12/CECs, and FI-S100A12-CECs in patients with KD with CALs

\begin{tabular}{lcccc}
\hline & $n$ & $\begin{array}{c}\text { CECs/MNC } \\
(\%)\end{array}$ & $\begin{array}{c}\text { CECs-S100A12/ } \\
\text { CECs }(\%)\end{array}$ & FI-S100A12-CECs \\
\hline $\begin{array}{c}\text { A-KD with } \\
\text { CALs }\end{array}$ & 10 & $1.29 \pm 1.43^{*}$ & $67 \pm 18^{*}$ & $221 \pm 242^{*}$ \\
$\begin{array}{c}\text { SA-KD with } \\
\text { CALs }\end{array}$ & 10 & $1.34 \pm 1.36^{*}$ & $60 \pm 25^{*}$ & $340 \pm 288^{*} \dagger$ \\
$\begin{array}{c}\text { C-KD with } \\
\text { CALs }\end{array}$ & 10 & $0.77 \pm 0.68 \ddagger$ & $58 \pm 20^{*}$ & $158 \pm 157^{*} \neq$ \\
Control & 60 & $0.69 \pm 0.50$ & $38 \pm 16$ & $61 \pm 50$ \\
\hline
\end{tabular}

$* p<0.05$ compared with control.

$\dagger p<0.05$ compared with A-KD.

$\ddagger p<0.05$ compared with A-KD and SA-KD.

$\mathrm{CECs} / \mathrm{MNC}$, the ratio of circulating endothelial cells to mononuclear cells; CECs-S100A12/CECs, the positive rate of S100A12 on the surface of circulating endothelial cells; FI-S100A12-CECs, the fluorescence intensity of S100A12 on the surface of circulating endothelial cells. with CALs than in controls. The CECs-S100A12/CECs rate did not decrease significantly in patients with SA-KD and C-KD with CALs. The FI-S100A12-CECs level increased significantly in patients with SA-KD with CALs, and decreased significantly in patients with $\mathrm{C}-\mathrm{KD}$, but it was still higher in patients with $\mathrm{C}-\mathrm{KD}$ with CALs than in controls (Table 3).

The comparison of CECs/MNC, CECs-S100A12/CECs, and FI-S100A12-CECs levels in KD with and without CALs. The CECs/MNC, CECs-S100A12/CECs, and FI-S100A12CECs levels had no difference in patients with A-KD with or without CALs. The CECs/MNC ratio and FI-S100A12-CECs levels in patients with SA-KD with CALs were significantly $(p<0.05)$ higher than those in patients with SA-KD without CALs. The CECs-S100A12/CECs level showed no difference in patients with SA-KD with or without CALs. Although the CECs/MNC ratio and CECs-S100A12/CECs rate had no difference in patients with C-KD with or without CALs, the FI-S100A12-CECs level was significantly $(p<0.05)$ higher in patients with $\mathrm{C}-\mathrm{KD}$ with CALs than in $\mathrm{C}-\mathrm{KD}$ without CALs (Fig. 2).

\section{DISCUSSION}

In this study, it was found that the CECs/MNC ratio increased significantly in patients with A-KD and decreased to near normal levels in patients with SA-KD without CALs after IVIG therapy, but it remained at high levels in patients with SA-KD with CALs after IVIG therapy and decreased to near normal levels in patients with C-KD. These findings suggest that the detachment of vascular endothelial cells increases significantly in patients with A-KD, and continues for a longer time in patients with KD with CALs. Nakatani et al. (12) found that the amount of CECs in patients with SA-KD was significantly higher than that in patients with C-KD and healthy controls, respectively, similar to that in patients with A-KD. It was not completely consistent with the results in our study, which may be due to the different timing of blood collection or case groups. This study reveals that the increasing of CECs in peripheral blood reflects lesion of vascular endothelial cells in patients with KD.

The study found that the CECs-S100A12/CECs rate increased significantly in patients with KD with or without CALs, and
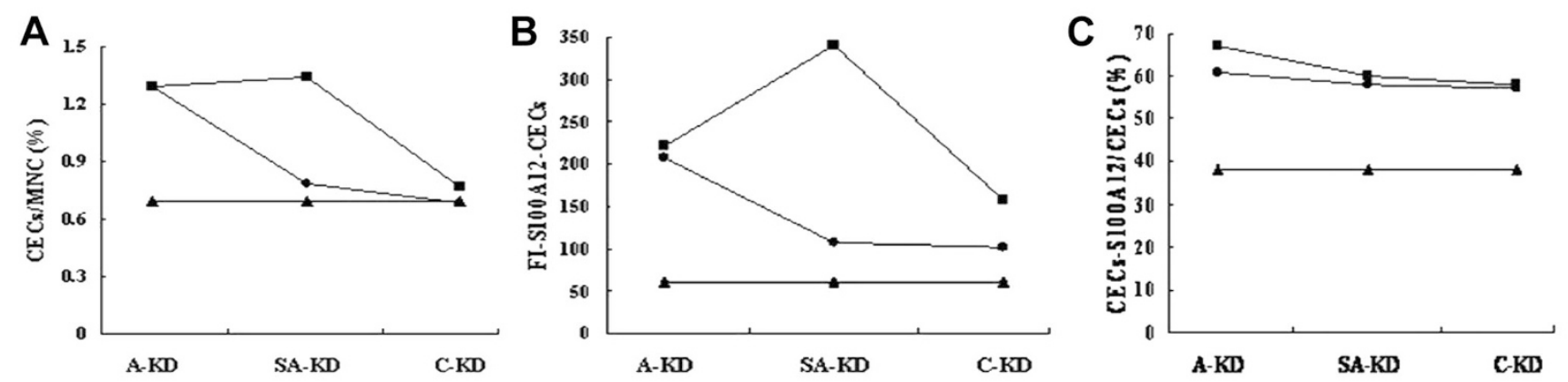

Figure 2. Diagram of CECs/MNC, CECs-S100A12/CECs and FI-S100A12-CECs levels in Kawasaki disease with or without coronary artery lesions. $A, \mathrm{CECs} / \mathrm{MNC}$ levels; B, CECs-S100A12/CECs levels; C, FI-S100A12-CECs levels. CECs/MNC, the ratio of circulating endothelial cells to MNC; CECs-S100A12/CECs, the positive rate of S100A12 on circulating endothelial cells surface; FI-S100A12-CECs, the fluorescence intensity of S100A12 on circulating endothelial cells surface; $\boldsymbol{\bullet}$ KD without CALs; $\mathbf{\square}$, KD with CALs; $\boldsymbol{\Delta}$, Control. 
decreased slowly in patients with SA-KD and C-KD, but it was still significantly higher in patients with $\mathrm{C}-\mathrm{KD}$ than in controls. Although the CECs/MNC ratio decreased to near normal levels in patients with SA-KD without CALs and in patients with $\mathrm{C}-\mathrm{KD}$ with CALs, the CECs-S100A12/CECs rate remained high in patients with SA-KD and C-KD, without significant decrease compared with that in patients with A-KD. The FI-S100A12CECs increased significantly in patients with A-KD, and decreased to near half levels in patients with SA-KD and C-KD without CALs, but it was still significantly higher in patients with C-KD than in controls. The FI-S100A12-CECs level increased continuously in patients with SA-KD with CALs and decreased in patients with $\mathrm{C}-\mathrm{KD}$, but it was still significantly higher in patients with C-KD than in controls. Ebihara et al. (15) found that the level of S100A12 protein in the peripheral blood of patients with A-KD was significantly higher than that in patients with SA-KD, C-KD and healthy controls, respectively, which suggests that the vascular endothelial cells and S100A12 protein participate in the development of $\mathrm{KD}$, but the mechanism is still unclear. This study indicates that the S100A12 expression on the CECs surface increased significantly in patients with $\mathrm{KD}$ and persisted for a longer time in patients with CALs, suggesting that the S100A12 expression on CECs may be involved in the development of CALs.

This study showed that the CECs/MNC rate decreased to near normal levels in patients with SA-KD without CALs after IVIG therapy, which confirms that IVIG therapy can reduce the CECs to normal levels probably by inhibiting inflammatory factors attacking the vascular endothelium and reducing the detachment of vascular endothelial cells. Ichivama et al. (20) demonstrated that IVIG could inhibit NF- $\kappa \mathrm{B}$ activation in coronary artery endothelial cells by regulating IL- 6 production and E-selecting expression, although the mechanism by which IVIG prevents CALs is still unknown. In patients with $\mathrm{KD}$ with CALs, the CECs/MNC rate kept at high levels in the SA-KD group after IVIG therapy, and decreased to near normal levels in the $\mathrm{C}-\mathrm{KD}$, these results suggesting that some patients are IVIG-resistant and the detachment of vascular endothelial cells may continue for a longer time.

The level of CECs decreased obviously in patients with SA-KD without CALs or C-KD patients with CALs, whereas the level of S100A12 protein on CECs did not reduce in patients with SA-KD and C-KD, which suggests that there may be continuous inflammatory reaction in the detached or undetached vascular endothelial cells attacked by S100A12, and this inflammation might last for over 2 months or even longer. Foell et al. (16) and Ye et al. (17) showed that the level of S100A12 decreased significantly after IVIG treatment, but Ye et al. measured the level of S100A12 in neutrophils while Foell et al. evaluated the S100A12 concentration in plasma, both of which are different from the measurement of CECsS100A12/CECs and FI-S100A12-CECs in this study. Our study demonstrates that S100A12 protein has strong ability to stick to the detached CECs in patients with SA-KD and C-KD, and it may be involved in the process of CECs damage, break, elimination, and apoptosis. IVIG therapy cannot improve inflammatory reaction caused by S100A12 protein in detached endothelial cells. S100A12 protein in peripheral blood is actively secreted by neutrophils, which cannot represent the level of S100A12 protein on CECs. The values of S100A12 protein in peripheral blood and on CECs have different meanings in patients with KD. The elevated level of S100A12 in peripheral blood signifies the level of inflammatory reaction in patients with KD. After IVIG treatment, the decreased expression of S100A12 in neutrophils or peripheral blood partly reduces the attack of endothelial cells caused by S100A12. However, IVIG therapy cannot completely suppress the inflammatory reaction caused by S100A12 protein on the endothelial cells, which is likely to clarify the mechanism that the CAL can be progressive when the inflammation index has returned to normal levels in some patients with KD.

\section{REFERENCES}

1. Cuttica RJ 1997 Vasculitis, Kawasaki disease, and pseudovasculitis. Curr Opin Rheumatol 9:448-457

2. Rowley AH, Shulman ST 1998 Kawasaki syndrome. Clin Microbiol Rev 11:405-414

3. Taubert KA, Rowley AH, Shulman ST 1991 Nationwide survey of Kawasaki disease and acute rheumatic fever. J Pediatr 119:279-282

4. Suzuki A, Kamiya T, Arakaki Y, Kinoshita Y, Kimura K 1994 Fate of coronary arterial aneurysms in Kawasaki disease. Am J Cardiol 74:822-824

5. Fujiwara T, Fujiwara H, Nakano H 1988 Pathological features of coronary arteries in children with Kawasaki disease in which coronary arterial aneurysm was absent at autopsy. Circulation 78:345-350

6. Fulton DR, Newburger JW 2000 Long-term cardiac sequelae of Kawasaki disease. Curr Rheumatol Rep 2:324-329

7. Kato H, Sugimura T, Akagi T, Sato N, Hashino K, Maeno Y, Kazue T, Eto G, Yamakawa R 1996 Long-term consequences of Kawasaki disease. A 10- to 21-year follow-up study of 594 patients. Circulation 94:1379-1385

8. Leung DY, Schlievert PM, Meissner HC 1998 The immunopathogenesis and management of Kawasaki syndrome. Arthritis Rheum 41:1538-1547

9. Nakamura Y, Yanagawa H, Harada K, Kato H, Kawasaki T 2000 Mortality among persons with a history of Kawasaki disease in Japan: existence of cardiac sequelae elevated the mortality. J Epidemiol 10:372-375

10. Newburger JW, Takahashi M, Gerber MA, Gewitz MH, Tani LY, Burns JC, Shulman ST, Bolger AF, Ferrieri P, Baltimore RS, Wilson WR, Baddour LM, Levison ME, Pallasch TJ, Falace DA, Taubert KA; Committee on Rheumatic Fever, Endocarditis and Kawasaki Disease; Council on Cardiovascular Disease in the Young; American Heart Association; American Academy of Pediatrics 2004 Diagnosis, treatment, and long-term management of Kawasaki disease: a statement for health professionals from the Committee on Rheumatic Fever, Endocarditis and Kawasaki Disease, Council on Cardiovascular Disease in the Young, American Heart Association. Circulation 110:2747-2771

11. Koc M, Richards HB, Bihorac A, Ross EA, Schold JD, Segal MS 2005 Circulating endothelial cells are associated with future vascular events in hemodialysis patients. Kidney Int 67:1078-1083

12. Nakatani K, Takeshita S, Tsujimoto H, Kawamura Y, Tokutomi T, Sekine I 2003 Circulating endothelial cells in Kawasaki disease. Clin Exp Immunol 131:536-540

13. Mikkelsen SE, Novitskaya V, Kriajevska M 2001 S100A12 protein is a strong inducer of neurite outgrowth from primary hippocampal neurons. J Neurochem 79:767-776

14. Miranda LP, Tao T, Jones A, Chernushevich I, Standing KG, Geczy CL, Alewood PF 2001 Total chemical synthesis and chemotactic activity of human S100A12 (EN-RAGE). FEBS Lett 488:85-90

15. Ebihara T, Endo R, Kikuta H, Ishiguro N, Ma X, Shimazu M, Otoguro T, Kobayash K 2005 Differential gene expression of S100 protein family in leukocytes from patients with Kawasaki disease. Eur J Pediatr 164:427-431

16. Foell D, Ichida F, Vogl T, Yu X, Chen R, Miyawaki T, Sorg C, Roth J 2003 S100A12 (EN-RAGE) in monitoring Kawasaki disease. Lancet 361:1270-1272

17. Ye F, Foell D, Hirono KI, Vogl T, Rui C, Yu X, Watanabe S, Watanabe K, Uese K, Hashimoto I, Roth J, Ichida F, Miyawaki T 2004 Neutrophil-derived S100A12 is profoundly upregulated in the early stage of acute Kawasaki disease. Am J Cardiol 94:840-844

18. Ayusawa M, Sonobe T, Uemura S, Ogawa S, Nakamura Y, Kiyosawa N, Ishii M, Harada K, Kawasaki Disease Research Committee 2005 Revision of diagnostic guidelines for Kawasaki disease (the 5th revised edition). Pediatr Int 47:232-234

19. Khan SS, Solomon MA, McCoy JP Jr 2005 Detection of circulating endothelial cells and endothelial progenitor cells by flow cytometry. Cytometry B Clin Cytom 64:1-8

20. Ichiyama T, Ueno Y, Isumi H, Niimi A, Matsubara T, Furukawa S 2004 An immunoglobulin agent (IVIG) inhibits NF-kappaB activation in cultured endothelial cells of coronary arteries in vitro. Inflamm Res 53:253-256 